\title{
QUANTUM SITE PERCOLATION ON AMENABLE GRAPHS
}

\author{
IVAN VESELIĆ
}

\begin{abstract}
We consider the quantum site percolation model on graphs with an amenable group action. It consists of a random family of Hamiltonians. Basic spectral properties of these operators are derived: non-randomness of the spectrum and its components, existence of an self-averaging integrated density of states and an associated trace-formula.
\end{abstract}

\section{Introduction: The Quantum Percolation model}

The quantum percolation model (QPM) consist of two building blocks which are both well studied in physics of disordered media.

Let us first introduce the classical site percolation model. It is used to model the flow of liquid through porous media, the spreading of forest fires or of diseases etc. Consider the graph $\mathbb{Z}^{d}$ where two vertices are connected by an edge if their Euclidean distance equals one. Equip each vertex $v \in \mathbb{Z}^{d}$ with a random variable $q(v)$ taking the value 0 with probability $p$ and " $\infty$ " with probability $1-p$ and being independent of the random variables at all other vertices. For each configuration of the randomness $\omega \in \Omega$ let $V(\omega):=\left\{v \in \mathbb{Z}^{d} \mid q(v)=0\right\}$. The percolation problem consists in studying the properties of connected components - called clusters of $V(\omega)$. Typical questions are: With what probability do infinite clusters exist? What is the average vertex number or diameter of a cluster? What is the probability that $0, v \in \mathbb{Z}^{d}$ are in the same cluster, etc.? One of the central results of percolation theory is the existence of a critical probability $p_{c}$, such that for $p>p_{c}$ (respectively for $p<p_{c}$ ) an infinite cluster exists (respectively does not exist) almost surely. See e.g. $[11,10]$ and the literature cited there.

Random lattice Hamiltonians are used to describe the motion of waves in disordered media. Each of them is a family of operators on $l^{2}\left(\mathbb{Z}^{d}\right)$ indexed by elements of a probability space. The family obeys an equivariance relation with respect to the action of a group. More precisely, the group acts in a consistent way on $l^{2}\left(\mathbb{Z}^{d}\right)$ by translations and on the probability space by ergodic, measure preserving transformations.

The spectral features of these random operators allow one to draw conclusions about the transport and wave-spreading properties of the modelled medium. Monograph expositions of this topic can be found in $[4,16,19]$.

2000 Mathematics Subject Classification. 35J10,81Q10,82B43.

Key words and phrases. integrated density of states, random Schrödinger operators, random graphs, site percolation.

30th October 2018. 
Let us define the simplest QPM: Let

$$
\left(A_{\omega} f\right)(v)=\sum_{\operatorname{dist}(v, w)=1} f(w) \quad \text { for all } v, w \in V(\omega) \text { and all } f \in l^{2}(V(\omega))
$$

be the adjacency operator of $V(\omega)$ introduced above. More precisely, $A_{\omega}$ is the adjacency operator of the induced sub-graph of $\mathbb{Z}^{d}$ with vertex set $V(\omega)$. Here "dist" denotes the distance function on this graph.

At this point let us explain why we chose $\infty$ as one of the values the random variable $q(v)$ takes. The adjacency operator on $\mathbb{Z}^{d}$ corresponds (up to an energy shift) to the kinetic energy part of a quantum Hamiltonian on the lattice. In this picture $q$ corresponds to the potential energy. In the quantum percolation model it vanishes on some sites, on others it is infinitely high, i.e. forms an impenetrable barrier for the quantum wave function.

The interesting feature of the QPM is that it defines a Laplacian on random geometry. More precisely, its domain of definition $l^{2}(V(\omega))$ varies with $\omega$. This is the main difference to the random lattice operators considered in $[4,16]$. After an extension of the notion of random lattice Hamiltonians the QPM fits in this framework. In our approach we rely on methods from [17, 14, 13], developed there to study operators on manifolds.

The QPM was first studied in $[8,7]$. There it was considered as a quantum mechanical model for electron-propagation in binary alloys where only one of the two types of atoms participates in the spreading of the wavepacket. The model attracted special attention because of the existence of molecular states, i.e. eigenvectors supported on finite regions of the infinite cluster, see $[12,5]$. The last cited reference is the motivation of the present paper and our results can be seen as a mathematically rigorous version of some arguments in [5] and their extension to more general graphs.

The integrated density of states (IDS) of a Hamiltonian is the number of eigenvalues per unit volume below a certain energy value. Thanks to the stationarity and ergodicity assumptions it is well defined for random Hamiltonians. The IDS of a random Hamiltonian captures its global spectral features and its understanding is the prerequisite of the study of finer spectral properties. In the present work we analyze this quantity and provide therewith a basis for a further study of the QPM, cf. Section 4 and [20].

The next section states the results of this note, Section 2 is devoted to their proofs and the last section concludes with a discussion of further research topics.

\section{Results: Spectral properties of finite Range hopping operators}

To describe the geometric setting we are working in precisely, let us recall basic notions from graph theory and fix the notation along the way. A graph $G=(V, E)$ is given by a set of vertices $V=V(G)$ and a set of edges $E=E(G) \subset(V \times V \backslash\{(v, v) \mid$ $v \in V\}) / \sim$. Here $\sim$ denotes the relation $(v, w) \sim(w, v)$. If $e=(v, w) \in E$, we call $v, w \in V$ nearest neighbours and endpoints of the edge $e$. By our definition a graph is simple: it contains neither multiple edges nor self-loops, i.e. edges joining a vertex to itself.

A path (of length $n$ ) in $G$ is an alternating sequence of vertices and edges $\left\{v_{0}, e_{1}, v_{1}, \ldots e_{n}, v_{n}\right\}$ such that $e_{j}=\left(v_{j-1}, v_{j}\right)$ for all $j=1, \ldots, n$. If there is a path between two vertices $v$ and $w$ they are called (path) connected. This relation 
partitions the graph into (path connected) components. If a component contains a infinite number of distinct vertices we call it an infinite component. The distance between two vertices $v, w \in V$ is defined by

$$
\begin{aligned}
\operatorname{dist}_{G}(v, w):=\operatorname{dist}(v, w) & \\
& :=\min \{\text { length of } p \mid p \text { is a path connecting } v \text { and } w\}
\end{aligned}
$$

Note that the distance between $v$ and $w$ in a sub-graph of $G$ may be larger than their distance in the original graph $G$. The vertex degree $\operatorname{deg}(v)$ of a vertex $v \in V$ equals the number of edges $e \in E$, such that $v$ is an endpoint of $e$.

Let $G$ and $G^{\prime}$ be graphs. A map $\phi: G \rightarrow G^{\prime}$ is called a graph-map or graphhomomorphism, if $\phi: V(G) \rightarrow V\left(G^{\prime}\right), \phi: E(G) \rightarrow E\left(G^{\prime}\right)$ and if for any $e=(v, w) \in$ $E(G)$, the image $\phi(e)$ equals $(\phi(v), \phi(w))$. A graph-map $\phi: G \rightarrow G$ which has an inverse graph-map is called a graph-automorphism or simply automorphism of $G$.

Let $\Gamma$ be a group of graph-automorphism acting on a graph $X$. It induces a projection map proj: $X \rightarrow X / \Gamma$. We assume that the quotient is a finite graph. This implies in particular that the degree of the vertices in $V$ is uniformly bounded. We denote the smallest upper bound by $\operatorname{deg}_{+}$. Chose a vertex $[v] \in V(X / \Gamma)$ and a representative $v \in[v] \subset V(X)$. Starting form $v$, lift pathwise the vertices and edges of $X / \Gamma$ to obtain a connected set of vertices and edges $\tilde{\mathcal{F}} \subset X$, such that $\left.\operatorname{proj}\right|_{\tilde{\mathcal{F}}}: \tilde{\mathcal{F}} \rightarrow X / \Gamma$ is a bijective map. The set $\mathcal{F}:=\tilde{\mathcal{F}} \cup\{v \in V(X) \mid v$ is an endpoint of an edge in $\mathcal{F}\}$ is a graph, which we call fundamental domain. Note that proj $\left.\right|_{\mathcal{F}}: \mathcal{F} \rightarrow X / \Gamma$ is a graph-map, which is bijective on the set of edges, but not on the set of vertices.

We construct a probability space $(\Omega, \mathcal{A}, \mathbb{P})$ associated to percolation on $X$. Let $\Omega=\times_{v \in V}\{0, \infty\}$ be equipped with the $\sigma$-algebra $\mathcal{A}$ generated by finite dimensional cylinders sets. Denote by $\mathbb{P}$ a probability measure on $\Omega$ and assume that the measurable shift transformations

$$
\tau_{\gamma}: \Omega \rightarrow \Omega, \quad\left(\tau_{\gamma} \omega\right)_{v}=\omega_{\gamma^{-1} v}
$$

are measure preserving. Moreover, let the family $\tau_{\gamma}, \gamma \in \Gamma$ act ergodically on $\Omega$. By the definition of $\tau_{\gamma}, \gamma \in \Gamma$ the stochastic field $q: \Omega \times V \rightarrow\{0, \infty\}$ given by $q(\omega, v)=\omega_{v}, v \in V$ is stationary or equivariant, i.e. $q\left(\tau_{\gamma} \omega, v\right)=q\left(\omega, \gamma^{-1} v\right)$. An element $\omega$ of the probability space will be called configuration. The mathematical expectation associated to the probability $\mathbb{P}$ will be denoted by $\mathbb{E}$.

For a configuration $\omega$, a site $v$ with $q(\omega, v)=0$ will be called active or undeleted and a site $v$ with $q(\omega, v)=\infty$ deleted.

For each $\omega \in \Omega$ denote by

$$
V(\omega)=V(X(\omega))=\{v \in V \mid q(\omega, v)=0\}
$$

the subset of active vertices, and denote by $X(\omega)$ the corresponding induced subgraph of $X$. It is the sub-graph of $X$ whose vertex set is $V(\omega)$ and whose edge set is

$$
E(\omega)=E(X(\omega))=\{e \in E(X) \mid \text { both endpoints of } e \text { are in } V(\omega)\}
$$

Let $\Lambda=(V(\Lambda), E(\Lambda))$ be an (deterministic) induced sub-graph of $X$. It gives rise to a random family of induced sub-graphs $\Lambda(\omega):=X(\omega) \cap \Lambda$.

On any of the graphs introduced so far we will consider operators of finite hopping range. The easiest example to have in mind is the adjacency operator $A_{\omega}$ considered 
already in (1). More generally, a operator of finite hopping range $H$ on a graph $G$ is a linear map $H: l^{2}(V(G)) \rightarrow l^{2}(V(G))$ such that there exists $C, R \leq \infty$ with

(i) $H(v, w)=H(w, v)$

(ii) $H(\gamma v, \gamma w)=H(v, w)$ for all $\gamma \in \Gamma$

(iii) $|H(v, w)| \leq C$ and

(iv) $H(v, w)=0$ if $\operatorname{dist}(v, w) \geq R$

for all $v, w \in V(G)$. Here $H(v, w):=\left\langle\delta_{v}, H \delta_{w}\right\rangle$ and $\delta_{v} \in l^{2}(V(G))$ is the function taking the value 1 at $v$ and 0 elsewhere.

For a sub-graph $G \subset X$ and a finite hopping range operator $H$ denote by $H^{G}$ the compression of $H$ to $l^{2}(V(G))$, in other words

$$
H^{G}(v, w)=H(v, w) \text { if } v, w \in G \text { and } H^{G}(v, w)=0 \text { otherwise }
$$

If $V=V(G)$ is finite, $H$ is a $(|V| \times|V|)$-matrix, where $|\cdot|$ denotes the cardinality of a set. Thus the spectrum of $H^{G}$ is real and consists entirely of eigenvalues $\lambda_{i}\left(H^{G}\right)$, which we enumerate in increasing order counting multiplicity. Let us define the normalized eigenvalue counting function of $H^{G}$ as

$$
N^{G}(H, \lambda):=\frac{\left|\left\{i \in \mathbb{N} \mid \lambda_{i}\left(H^{G}\right)<\lambda\right\}\right|}{|V|}
$$

We assume that the discrete group $\Gamma$ is amenable, i.e. there exists a $\mathrm{F} \varnothing \operatorname{lner}$ sequence $\left\{I_{j}\right\}_{j}$ of finite, non-empty subsets of $\Gamma$. A sequence $\left\{I_{j}\right\}_{j}$ is called Følner sequence if for any finite $K \subset \Gamma$ and $\epsilon>0$

$$
\left|I_{j} \Delta K I_{j}\right| \leq \epsilon\left|I_{j}\right|
$$

for all $j$ large enough. Since the quotient $X / \Gamma$ is compact, it follows that $K:=\{\gamma \in$ $\Gamma \mid \gamma \mathcal{F} \cap \mathcal{F} \neq \emptyset\}$ is a finite generator set for $\Gamma$, cf. $\S 3$ in [2] for a similar statement in the context of manifolds. Now for finitely generated amenable groups there exists a Følner sequence of subsets, which is increasing and exhausts $\Gamma$, cf. Theorem 4 in [1]. From [15] we infer that each Følner sequence has an tempered subsequence. A tempered $\mathrm{F} \varnothing$ lner sequence is a sequence which satisfies in addition to (3) the growth condition

$$
\text { there exists } C<\infty \text { such that for all } j \in \mathbb{N}:\left|I_{j} I_{j-1}^{-1}\right| \leq C\left|I_{j}\right|
$$

To each increasing, tempered Følner sequence associate an admissible exhaustion $\left\{\Lambda_{j}\right\}_{j}$ of $X$ given by

$$
\Lambda_{j}:=\bigcup_{\gamma \in I_{j}^{-1}} \gamma \mathcal{F} \subset X
$$

where $I_{j}^{-1}:=\left\{\gamma \mid \gamma^{-1} \in I_{j}\right\}$

For a finite hopping range operator $H$, a Følner sequence $\left\{I_{j}\right\}_{j}$, and a random configuration $\omega \in \Omega$ introduce for brevity sake the following notation: $H_{\omega}:=$ $H^{X(\omega)}, H_{\omega}^{j}:=H^{\Lambda_{j}(\omega)}$, and $N_{\omega}^{j}(\lambda):=N\left(H_{\omega}^{j}, \lambda\right)$. Denote by $P_{\omega}(I):=\chi_{I}\left(H_{\omega}\right)$ the spectral projection of $H_{\omega}$ associated to the energy interval $I$.

Theorem 2.1. There exist a distribution function $N$ called integrated density of states such that for almost all $\omega \in \Omega$ and any admissible exhaustion $\Lambda_{j}, j \in \mathbb{N}$ we have

$$
\lim _{j \rightarrow \infty} N_{\omega}^{j}(E)=N(E)
$$


at all continuity points of $N$. The following trace formula holds for the IDS

$$
N(E)=\frac{1}{|\mathcal{F}|} \mathbb{E}\left\{\operatorname{Tr}\left(\chi_{\mathcal{F}} P_{\omega}(]-\infty, E[)\right)\right\}
$$

We say that the IDS $N$ is associated to the sequence of random operators $\left\{H_{\omega}^{j}\right\}_{\omega \in \Omega}, j \in \mathbb{N}$. Next we address the question of boundary condition dependence. Denote $\Lambda^{c}=X \backslash \Lambda$.

Proposition 2.2. Let $H$ be a finite hopping range operator, $\Lambda_{j}, j \in \mathbb{N}$ an admissible exhaustion and $\tilde{R} \in \mathbb{N}, C<\infty$. Let $B^{j}: l^{2}\left(\Lambda_{j}\right) \rightarrow l^{2}\left(\Lambda_{j}\right), j \in \mathbb{N}$ be any sequence of symmetric operators such that for all $v, w \in V$ we have $\left|B^{j}(v, w)\right| \leq C$ and $B^{j}(v, w)=0$ if $\operatorname{dist}\left(v, \Lambda_{j}^{c}\right)+\operatorname{dist}\left(w, \Lambda_{j}^{c}\right)>\tilde{R}$. Then the IDS' associated to the sequences $\left\{H_{\omega}^{j}\right\}_{\omega \in \Omega}, j \in \mathbb{N}$ and $\left\{H_{\omega}^{j}+B^{j}\right\}_{\omega \in \Omega}, j \in \mathbb{N}$ coincide.

Next we establish the non-randomness of the spectrum of $H_{\omega}$ and its components, its relation to the IDS and an understanding of the IDS as a von Neumann trace. Denote by $\sigma_{d i s c}, \sigma_{e s s}, \sigma_{a c}, \sigma_{s c}, \sigma_{p p}$ the discrete, essential, absolutely continuous, singular continuous, and pure point part of the spectrum. Denote by $\sigma_{\text {comp }}$ the set of eigenvalues which posses an eigenfunction with compact, i.e. finite, support. In the following theorem $\Gamma$ need not be amenable, but $X$ must be countable.

Theorem 2.3. There exists a $\Omega^{\prime} \subset \Omega$ of full measure and subsets of the real numbers $\Sigma$ and $\Sigma_{\bullet}$, where $\bullet \in\{$ disc, ess, ac, sc,pp, comp $\}$, such that for all $\omega \in \Omega^{\prime}$

$$
\sigma\left(H_{\omega}\right)=\Sigma \quad \text { and } \quad \sigma_{\bullet}\left(H_{\omega}\right)=\Sigma \bullet
$$

for any $\bullet=$ disc, ess, ac, sc,pp, comp. If $\Gamma$ is infinite, $\Sigma_{\text {disc }}=\emptyset$. The almost-sure spectrum $\Sigma$ coincides with the set of points of increase of the IDS

$$
\Sigma=\{\lambda \in \mathbb{R} \mid N(\lambda+\epsilon)>N(\lambda-\epsilon) \text { for all } \epsilon>0\}
$$

Furthermore, $N$ is the distribution function of the spectral measure of the direct integral operator

$$
\mathcal{H}:=\int_{\Omega}^{\oplus} H_{\omega} d \mathbb{P}(\omega)
$$

On the von Neumann algebra associated to $\mathcal{H}$ there is a canonical trace and $N(E)$ is the value of this trace on the spectral projection of $\mathcal{H}$ associated to the interval ]$-\infty, E[$.

\section{Proofs of the theOrems}

Let $H$ be a finite hopping range operator and assume without loss of generality $|H(v, w)| \leq 1$ for all matrix elements. It follows that the $l^{2}$-norm of $H$ is bounded by $K:=2 \operatorname{deg}_{+}^{R}$. Since $H$ is symmetric, it is a selfadjoint operator. In particular the spectrum of $H_{\omega}$ is contained in $[-K, K]$ for all $\omega \in \Omega$.

Each $\gamma \in \Gamma$ induces an unitary operator $U_{\omega, \gamma}: l^{2}\left(V\left(\tau_{\gamma}^{-1} \omega\right)\right) \rightarrow l^{2}(V(\omega)),\left(U_{\omega, \gamma} f\right)(v):=$ $f\left(\gamma^{-1} v\right)$. Note that $V\left(\tau_{\gamma} \omega\right)=\gamma V(\omega)$. By the definition of $\tau_{\gamma}$ the action of $\Gamma$ on $\Omega$ and on $X$ is compatible:

$$
U_{\omega, \gamma} H_{\omega} U_{\omega, \gamma}^{*}=H_{\tau_{\gamma} \omega}
$$

The equivariance formula (5) implies

$$
U_{\omega, \gamma} f\left(H_{\omega}\right) U_{\omega, \gamma}^{*}=f\left(H_{\tau_{\gamma} \omega}\right)
$$


for any polynomial $f$. For continuous functions $f, g$ we have $\left\|f\left(H_{\omega}\right)-g\left(H_{\omega}\right)\right\| \leq$ $\|f-g\|_{\infty}$. Thus $f_{n} \rightarrow f$ in $C\left([-K, K],\|\cdot\|_{\infty}\right)$ implies $f_{n}\left(H_{\omega}\right) \rightarrow f\left(H_{\omega}\right)$ in operator norm, and (6) extends by Weierstraß' approximation theorem to all $f \in$ $C([-K, K])$. By taking scalar products we obtain the corresponding equivariance relation for the matrix elements:

$$
f\left(H_{\omega}\right)\left(\gamma^{-1} v, \gamma^{-1} w\right)=f\left(H_{\tau_{\gamma} \omega}\right)(v, w)
$$

For the proof of the main Theorem 2.1 we need two key ingredients: an estimate of boundary effects on traces and a sufficiently general ergodic theorem, which will be applied to trace functionals of the type

$$
F(\omega):=|\mathcal{F}|^{-1} \sum_{v \in \mathcal{F}} f\left(H_{\omega}\right)(v, v)=|\mathcal{F}|^{-1} \operatorname{Tr}\left(f\left(H_{\omega}\right) \chi_{\mathcal{F}}\right)
$$

Let us first estimate the boundary effects.

Proposition 3.1. Let $f(x)=x^{m}$ for $m \in \mathbb{N}$. Then

$$
\sup _{\omega \in \Omega} \frac{1}{\left|\Lambda_{j}\right|}\left|\operatorname{Tr}\left(f\left(H_{\omega}^{j}\right)\right)-\operatorname{Tr}\left(\chi_{\Lambda_{j}} f\left(H_{\omega}\right)\right)\right| \rightarrow 0
$$

as $j \rightarrow \infty$.

Proof. We introduce the notion of a thickened boundary on a graph. For a subgraph $\Lambda$ and $h \in \mathbb{N}$ set $\partial_{h} \Lambda:=\left\{v \in \Lambda \mid \operatorname{dist}\left(v, \Lambda^{c}\right) \leq h\right\}$. We expand the trace of powers of $H_{\omega}^{j}$ :

$$
\operatorname{Tr}\left(H_{\omega}^{j}\right)^{m}=\sum_{v \in \Lambda_{j}}\left(H_{\omega}^{j}\right)^{m}(v, v)=\sum_{v \in \Lambda_{j}} \sum_{v_{1}, \ldots, v_{m-1} \in \Lambda_{j}} H_{\omega}\left(v, v_{1}\right) \ldots H_{\omega}\left(v_{m-1}, v\right)
$$

By an analogous formula for $\operatorname{Tr}\left(\chi_{\Lambda_{j}} H_{\omega}^{m}\right)$ we obtain

$$
\begin{array}{r}
\operatorname{Tr}\left[\chi_{\Lambda_{j}} H_{\omega}^{m}-\left(H_{\omega}^{j}\right)^{m}\right]=\sum_{v \in \Lambda_{j}} \sum_{\bullet} H_{\omega}\left(v, v_{1}\right) \ldots H_{\omega}\left(v_{m-1}, v\right) \\
\leq\left|\partial_{R m} \Lambda_{j}\right| \operatorname{deg}_{+}^{m^{2} R}
\end{array}
$$

where the bullet denotes summation over $m$-1-tuples (paths) in $V(X)$ with at least one vertex outside $\Lambda_{j}$. By the Følner property of the sequence $I_{j}, j \in \mathbb{N}$ we have:

$$
\lim _{j \rightarrow \infty} \frac{\left|\partial_{h} \Lambda_{j}\right|}{\left|\Lambda_{j}\right|}=0 \quad \text { for any } h \geq 0
$$

This is the content of Lemma 2.4 in [17]. In fact, there manifolds are considered, but the proof applies literally to the case of graphs.

Lindenstrauss proved in [15] a remarkable ergodic theorem which applies to locally compact, second countable, amenable groups. It includes the following statement for discrete groups.

Theorem 3.2. Let $\Gamma$ be an amenable discrete group and $\left(\Omega, \mathcal{B}_{\Omega}, \mathbb{P}\right)$ be a probability space. Assume that $\Gamma$ acts ergodically on $\Omega$ by measure preserving transformations $\tau_{\gamma}$. Let $\left\{I_{j}\right\}_{j}$ be a tempered Følner sequence in $\Gamma$. Then for every $F \in L^{1}(\Omega)$

$$
\lim _{j \rightarrow \infty} \frac{1}{\left|I_{j}\right|} \sum_{\gamma \in I_{j}} F\left(\tau_{\gamma} \omega\right)=\mathbb{E}\{F\}
$$

for almost all $\omega \in \Omega$. 
In the application we have in mind $F \in L^{\infty}$, so the convergence holds in the $L^{1}$-topology, too.

Proof of Theorem 2.1. To prove the claimed convergence of distribution functions it is sufficient to establish $\int f d N_{\omega}^{j} \rightarrow \int f d N$ for all $f \in C([-K, K])$. This can in turn be reduced to proving the convergence in the case where $f$ is a polynomial: By Weierstraß' approximation theorem the polynomials are dense in $C([-K, K])$. Let $f_{k}$ be a sequence of polynomials such that $\left\|f_{k}-f\right\|_{\infty} \rightarrow 0$. For any $\epsilon>0$ one can choose $k$ large enough such that $\left\|f_{k}-f\right\|_{\infty}<\epsilon / 3$ and subsequently $j$ large enough (depending on $\epsilon$ and $f_{k}$ ) such that $\left|\int f_{k} d N_{\omega}^{j}-\int f_{k} d N\right|<\epsilon / 3$. Since the total measure of $N$ and any $N_{\omega}^{j}$ is bounded by one,

$$
\begin{aligned}
\mid \int f d N_{\omega}^{j}- & \int f d N \mid \\
\leq & \left|\int\left(f-f_{k}\right) d N_{\omega}^{j}\right|+\left|\int f_{k} d N_{\omega}^{j}-\int f_{k} d N\right|+\left|\int\left(f_{k}-f\right) d N\right|<\epsilon
\end{aligned}
$$

Thus it is sufficient to prove the convergence of moments

$$
\lim _{j \rightarrow \infty} \int_{\mathbb{R}} \lambda^{m} N_{\omega}^{j}(d \lambda)=\int_{\mathbb{R}} \lambda^{m} N(d \lambda) \quad \text { for all } m \in \mathbb{N}
$$

Next we show that the limit on the left hand side equals $\frac{1}{|\mathcal{F}|} \mathbb{E}\left\{\operatorname{Tr}\left(\chi_{\mathcal{F}} H_{\omega}^{m}\right)\right\}$ for almost all $\omega$. For this aim we write the moment of the IDS as a trace

$$
\int_{\mathbb{R}} \lambda^{m} N_{\omega}^{j}(d \lambda)=\left|\Lambda_{j}\right|^{-1} \operatorname{Tr}\left(f\left(H_{\omega}^{j}\right)\right)
$$

which by Proposition 3.1 converges for $j \rightarrow \infty$ to the same limit as $\left|\Lambda_{j}\right|^{-1} \operatorname{Tr}\left(\chi_{\Lambda_{j}} f\left(H_{\omega}\right)\right)$. Now we decompose the trace according to local contributions and apply Lindenstrauss' theorem

$$
\begin{aligned}
&\left|\Lambda_{j}\right|^{-1} \operatorname{Tr}\left(\chi_{\Lambda_{j}} f\left(H_{\omega}\right)\right)=\left|\Lambda_{j}\right|^{-1} \sum_{v \in \Lambda_{j}} f\left(H_{\omega}^{j}\right)(v, v) \\
&\left|\Lambda_{j}\right|^{-1} \sum_{\gamma \in I_{j}} \sum_{v \in \mathcal{F}} f\left(H_{\omega}\right)\left(\gamma^{-1} v, \gamma^{-1} v\right)=\left|I_{j}\right|^{-1} \sum_{\gamma \in I_{j}}|\mathcal{F}|^{-1} \sum_{v \in \mathcal{F}} f\left(H_{\tau_{\gamma} \omega}\right)(v, v) \\
& \rightarrow \mathbb{E}\{F\} \quad \text { as } j \rightarrow \infty \text { for almost all } \omega \in \Omega
\end{aligned}
$$

where $F(\omega)=|\mathcal{F}|^{-1} \sum_{v \in \mathcal{F}} f\left(H_{\omega}\right)(v, v)=|\mathcal{F}|^{-1} \operatorname{Tr}\left(\chi_{\mathcal{F}} f\left(H_{\omega}\right)\right)$. Set $E_{\omega}(\lambda):=P_{\omega}(]-\infty, \lambda[)$. The expectation of $F$ equals

$$
\begin{aligned}
& \frac{1}{|\mathcal{F}|} \mathbb{E}\left\{\sum_{v \in \mathcal{F}} \int \lambda^{m} E_{\omega}(d \lambda)(v, v)\right\} \\
&=\frac{1}{|\mathcal{F}|} \int \lambda^{m} \mathbb{E}\left\{\operatorname{Tr}\left(\chi_{\mathcal{F}} E_{\omega}(d \lambda)\right)\right\}=\int \lambda^{m} N(d \lambda)
\end{aligned}
$$


Proof of Proposition 2.2. Denote $\tilde{H}_{\omega}^{j}:=H_{\omega}^{j}+B^{j}$. Similarly as in the proof of Proposition 3.1 we have

$$
\begin{array}{r}
\operatorname{Tr}\left[\left(\tilde{H}_{\omega}^{j}\right)^{m}-\left(H_{\omega}^{j}\right)^{m}\right] \\
=\sum_{v \in \Lambda_{j}} \sum_{\bullet} H_{\omega}\left(v, v_{1}\right) \ldots H_{\omega}\left(v_{m-1}, v\right)-\tilde{H}_{\omega}\left(v, v_{1}\right) \ldots \tilde{H}_{\omega}\left(v_{m-1}, v\right) \\
\leq\left[1+(1+C)^{m}\right]\left|\partial_{R m+\tilde{R}_{j}}\right| \operatorname{deg}_{+}^{m^{2} r}
\end{array}
$$

Here the bullet denotes the summation over all paths $v_{1}, \ldots, v_{m-1} \in \Lambda_{j}$ with at least one vertex in $\partial_{\tilde{R}} \Lambda_{j}$ and $r:=\max (R, \tilde{R})$. By the Følner property $\left|\Lambda_{j}\right|^{-1} \operatorname{Tr}\left[\left(\tilde{H}_{\omega}^{j}\right)^{m}-\right.$ $\left.\left(H_{\omega}^{j}\right)^{m}\right]$ converges to zero as $j \rightarrow \infty$.

Proof of Theorem 2.3. First we prove the non-randomness of $\sigma_{\text {comp }}$. Set

$$
\begin{aligned}
\tilde{\Sigma}:= & \left\{E \in \mathbb{R} \mid \exists \text { finite induced sub-graph } G \subset X \text { and } f \in l^{2}(G)\right. \\
& \text { such that } \left.H^{G} f=E f\right\}
\end{aligned}
$$

Then $\sigma_{\text {comp }}\left(H_{\omega}\right) \subset \tilde{\Sigma}$ for all $\omega \in \Omega$. Since $X$ is countable, there exists a countable exhaustion of $X$ by finite sets $D_{j}, j \in \mathbb{N}$. If we set

$$
\tilde{\Sigma}_{j}:=\left\{E \in \mathbb{R} \mid \exists \omega \in \Omega \text { and } f \in l^{2}\left(D_{j}(\omega)\right) \text { s.t. } H^{D_{j}(\omega)} f=E f\right\},
$$

then $\tilde{\Sigma}=\cup_{j} \tilde{\Sigma}_{j}$ and thus $\tilde{\Sigma}$ is countable.

For any $E \in \tilde{\Sigma}$ set $\Omega_{E}:=\left\{\omega \mid \exists f\right.$ with finite support and $\left.H_{\omega} f=E f\right\}$. This set is invariant under the ergodic action of $\Gamma$ by the transformations $\tau_{\gamma}$. Therefore, either $\mathbb{P}\left(\Omega_{E}\right)=1$ or $\mathbb{P}\left(\Omega_{E}\right)=0$. In the first case set $\tilde{\Omega}_{E}=\Omega_{E}$ and $E \in \Sigma_{\text {comp }}$, in the second set $\tilde{\Omega}_{E}=\Omega_{E}^{c}$ and $E \notin \Sigma_{\text {comp }}$. Here the superscript ${ }^{c}$ denotes the complement of a set. The set $\tilde{\Omega}:=\cap_{E \in \tilde{\Sigma}} \tilde{\Omega}_{E}$ has full measure and each $\omega \in \tilde{\Omega}$ satisfies $\sigma_{\text {comp }}\left(H_{\omega}\right)=\Sigma_{\text {comp }}$.

The remaining statements of the theorem follow from the results of [13]. One just has to check that the required assumptions are satisfied. This is not hard, but it would require to introduce the notion of grupoids and basic features of Connes' non-commutative integration theory [6]. Therefore we leave the details of the proof of Theorem 2.3 to another occasion.

\section{Outlook: Finitely SUPPORTED AND EXPONENTIALLY DECAYING STATES}

Once we have a rigorous definition of the integrated density of states for the QPM, we can study finer spectral properties. One of the main interest in the physics literature are the properties of bound states, and their contribution to numerically observed "peaks" of the density of states. This quantity is the distributional derivative of the IDS. In the following we restrict our discussion to the QPM corresponding to the adjacency operator $A$ on the lattice $\mathbb{Z}^{d}$, and to the sequence $q(\cdot, v), v \in \mathbb{Z}^{d}$, consisting of independent, identically distributed random variables.

There seem to be three different types of bound states of the QPM: finite cluster states, molecular states and exponentially decaying states, cf. $[8,7,12,18,5]$. Finite cluster states occur since almost surely there are active clusters of finite size, which, consequently, can support only bound states. These states are mathematically not 
challenging.

However, as pointed out earlier, there exist molecular states. They are eigenvectors of the adjacency operator restricted to the active sites with support on a finite region of the infinite cluster. This means that due to the deletion of sites the unique continuation property of eigenfunctions of the adjacency operator on the lattice breaks down.

For the analysis of molecular states it is convenient to introduce the restriction $A_{\omega}^{\infty}$ of $A$ to the infinite active cluster $X^{\infty}(\omega)$, and to define the corresponding IDS by

$$
N^{\infty}(\lambda)=|\mathcal{F}|^{-1} \mathbb{E}\left\{\operatorname{Tr}\left[\chi_{\mathcal{F} \infty(\omega)} E_{\omega}^{\infty}(\lambda)\right]\right\}
$$

where $\mathcal{F}^{\infty}(\omega)=\mathcal{F} \cap X^{\infty}(\omega)$ and $\mathcal{F}=\{0\} . N^{\infty}$ is self-averaging, i.e. can be defined by an exhaustion procedure, similarly as $N$ in Theorem 2.1.

Here is a result on molecular states, whose proof is given in [20].

Theorem 4.1. The set of points of discontinuity of the IDS $N$ of $\left\{A_{\omega}\right\}_{\omega}$ coincides with the set of points of discontinuity of the IDS $N^{\infty}$ of $\left\{A_{\omega}^{\infty}\right\}_{\omega}$ and equals $\tilde{\Sigma}$ as defined in (9).

Finally, one can hope that a multi-scale argument will yield a proof of the existence of exponentially decaying eigenstates, as in the case of the Anderson model $[3,9,21,19]$. For this aim, one has to develop new tools to deal with the singular randomness present in the QPM. On the other hand, once this is done, one might use the new ideas to approach the exponential localization problem for the Anderson model with Bernoulli disorder of the coupling constants. In the multi-dimensional case this is a problem which is open since decades.

\section{REFERENCES}

[1] T. Adachi. A note on the Følner condition for amenability. Nagoya Math. J., 131:67-74, 1993.

[2] T. Adachi and T. Sunada. Density of states in spectral geometry. Comment. Math. Helv., 68(3):480-493, 1993.

[3] P. Anderson. Absence of diffusion in certain random lattices. Phys. Rev., 109:1492, 1958.

[4] R. Carmona and J. Lacroix. Spectral Theory of Random Schrödinger Operators. Birkhäuser, Boston, 1990.

[5] J. T. Chayes, L. Chayes, J. R. Franz, J. P. Sethna, and S. A. Trugman. On the density of states for the quantum percolation problem. J. Phys. A, 19(18):L1173-L1177, 1986.

[6] A. Connes. Sur la théorie non commutative de l'intégration. In Algèbres d'opérateurs (Sém., Les Plans-sur-Bex, 1978), pages 19-143. Springer, Berlin, 1979.

[7] P.-G. de Gennes, P. Lafore, and J. Millot. Amas accidentels dans les solutions solides désordonnées. J. of Phys. and Chem. of Solids, 11(1-2):105-110, 1959.

[8] P.-G. de Gennes, P. Lafore, and J. Millot. Sur un phénomène de propagation dans un milieu désordonné. J. Phys. Rad., 20:624, 1959.

[9] J. Fröhlich and T. Spencer. Absence of diffusion in the Anderson tight binding model for large disorder or low energy. Commun. Math. Phys., 88:151-184, 1983.

[10] G. Grimmett. Percolation, volume 321 of Grundlehren der Mathematischen Wissenschaften. Springer, Berlin, 1999.

[11] H. Kesten. Percolation theory for mathematicians, volume 2 of Progress in Probability and Statistics. Birkhäuser, Boston, 1982.

[12] S. Kirkpatrick and T. P. Eggarter. Localized states of a binary alloy. Phys. Rev. B, 6:3598, 1972.

[13] D. Lenz, N. Peyerimhoff, and I. Veselić. Integrated density of states for random metrics on manifolds. (arXiv.org, math-ph/0212058), to appear in Proc. London Math. Soc. 
[14] D. Lenz, N. Peyerimhoff, and I. Veselić. Von Neumann algebras, groupoids and the integrated density of states. (math-ph/0203026 on arXiv.org), submitted, March 2002.

[15] E. Lindenstrauss. Pointwise theorems for amenable groups. Invent. Math., 146(2):259-295, 2001.

[16] L. A. Pastur and A. L. Figotin. Spectra of Random and Almost-Periodic Operators. Springer Verlag, Berlin, 1992.

[17] N. Peyerimhoff and I. Veselić. Integrated density of states for ergodic random Schrödinger operators on manifolds. Geom. Dedicata, 91(1):117-135, 2002.

[18] Y. Shapir, A. Aharony, and A. B. Harris. Localization and quantum percolation. Phys. Rev. Lett., 49(7):486-489, 1982.

[19] P. Stollmann. Caught by disorder: A Course on Bound States in Random Media, volume 20 of Progress in Mathematical Physics. Birkhäuser, 2001.

[20] I. Veselić. Spectral Analysis of Percolation Hamiltonians. arXiv.org/math-ph/0405006, accepted for publication in Math. Ann..

[21] H. von Dreifus and A. Klein. A new proof of localization in the Anderson tight binding model. Commun. Math. Phys., 124:285-299, 1989.

Fakultät für Mathematik, D-09107 TU Chemnitz

E-mail address: ivan.veselic@mathematik.tu-chemnitz.de

$U R L:$ www.tu-chemnitz.de/mathematik/schroedinger/ 